\title{
Impact phenomena assessment: Part II - Buffer container as a measure to reduce cargo leakage in collision
}

\author{
Aditya Rio Prabowo ${ }^{1}$, Seung Jun Baek ${ }^{2}$, Seung Geon Lee $^{2}$, \\ Dong Myung Bae ${ }^{2}$, Jung Min Sohn ${ }^{1,2, *}$, Aldias Bahatmaka ${ }^{1}$ and Samuel Samuel ${ }^{2}$ \\ ${ }^{1}$ Pukyong National University, Marine Convergence Design, 48513 Busan, Korea \\ ${ }^{2}$ Pukyong National University, Naval Architecture and Marine Systems Eng., 48513 Busan, Korea
}

\begin{abstract}
The safety of ship under collision has been developed in terms of navigational and structural aspects. These efforts are aimed to provide more safety from the internal part of a ship. However, in a collision, the external dynamics also needs to be considered, for instance velocity and location. In this study, these parameters are applied and observed regarding on a series of ship-container collision scenario. A freight container is used in the first stage and behaviours such as internal energy and effective stress are summarized. An alternative design so called buffer container is presented to offer better protection to a liquid cargo. The second stage is started by applying similar collision scenario as the first stage to the buffer container and comparing structural behaviour of this analysis. It is obtained that leakage possibility can be reduced on the buffer design as the strength has become approximately twice better than the freight container.
\end{abstract}

\section{Introduction}

Rapid development of human race affects every branch of science and technology to fulfil various societies' demands, for example, cargo distribution from production center to both national and international markets. One of the most important thing is to keep cargos safe during the process. Shipping container is developed to carry out this mission which the container can be multi-purpose carrier including for fast-degradable commodities and dangerous fluids, such as oil and gas. During the distribution process, the container is mostly transported by ship since it is acceptable in terms of cost and time efficiencies. However, safety for the container could be threatened by a collision. On the sea, this phenomenon is possibly triggered by arrangement failure of container column on the container ship, which leads to containers fall down to sea and collides with the ship hull.

In this study, objective is determined to propose a new container concept which is called buffer container as a measure to reduce cargo leakage in collision. A freight container is considered as representative of the existed design which to be analysed with parameters of the external dynamic of ship collision, namely velocity and location. The buffer container is developed based on the analysis result of the freight container.

\footnotetext{
Corresponding author: jminz@pknu.ac.kr
} 


\section{Literature review}

The operational system of every engineering design has a risk for unexpected phenomena such as an accidental impact. This situation is taken seriously in field of marine structures which structural failure due to an impact may trigger further disastrous events. Among of all impact forms, a ship collision is ranked as top-three dangerous evens for maritime accident. Certain parties across the globe have paid their intention to this impact form. Zhang [1] proposed a mechanical expression to assess a structural response after a collision. These methodologies were re-conducted in recent researches by comparing the numerical method using finite element (FE) approach, such as presented by researchers from Indonesia [2], and South Korea [3]. In terms of freight container incidents since late 1990s until 2017, APL China is considered as the worst one when records up to 100 million US Dollars financial loss. Other casualties in forms of a damaged container and cargo lost are main concerns for this incident type. Most of container incidents, for instance container collapse of the CSAV Shenzen and a recent accident of San Rafael in Vigo, Spain, produced damaged container and cargo lost. Terrible chain reaction in form of marine pollution was also recorded during ship grounding of the MSC Napoli in South-West England. Condition of container cargo especially in a fluid-hazardous state has to be taken as priority.

Arrangement failure of a container ship may lead to collision with other marine structure, e.g. ship and rupture of container walls and internal cargo (such as drum which carries dangerous cargo) cannot be avoided. Worse situation may occur if the cargo gets spilled inside the container which may initiate explosion or other dangerous chain reaction. Considering demands to ensure cargo safety against impact load, an alternative design of a freight container to ensure hazardous materials are still well contained during spontaneous impacts on ship, is considered as a research opportunity and determined as the state-of-theart for this study. Impact analysis considers ship-container collision model as the ship is denoted as representative of marine structure. In modelling stage, a freight container is used as conventional model which will be analysed and developed. Several structural responses are used parameter to evaluate the containers against impact.

\section{Numerical models}

The double hull vessel is idealised with the Length $L=85.92 \mathrm{~m}$, Breadth $B=15 \mathrm{~m}$ and Depth $H=10.4 \mathrm{~m}$. In the finite element model, this ship is modelled as four node-shell elements with a deformable material and the hull part is applied with the high-strength lowalloy steel with Density $\rho=7850 \mathrm{~kg} / \mathrm{m}^{3}$, Young's modulus $E=210,000 \mathrm{MPa}$, Poisson's ratio $n=0.3$ and Yield strength $\sigma_{Y}=315 \mathrm{MPa}$.

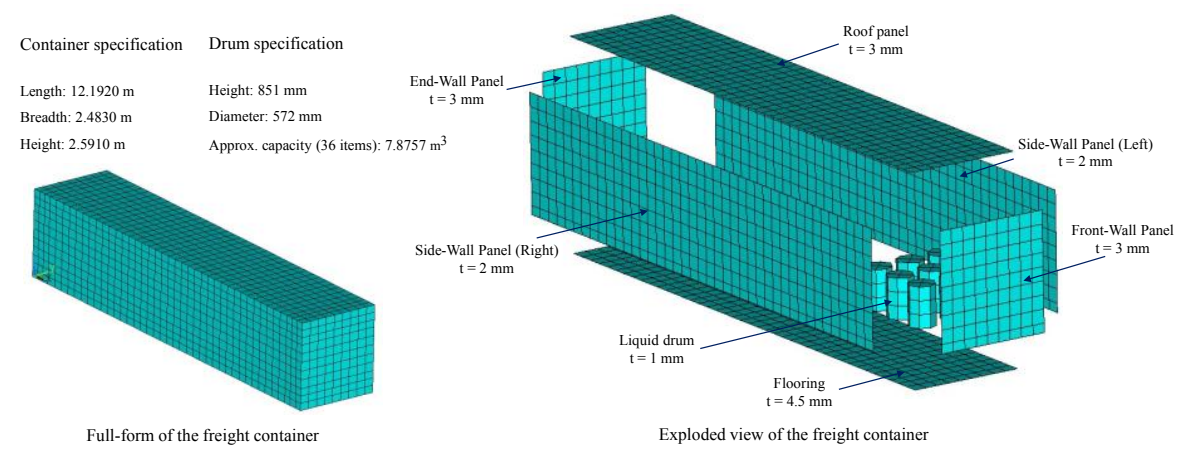

Fig. 1. Numerical model for the freight container. 


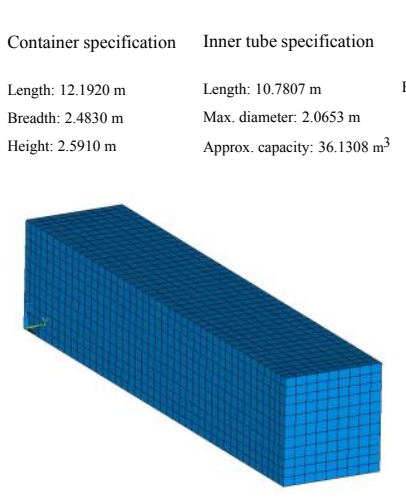

Full-form of the buffer container

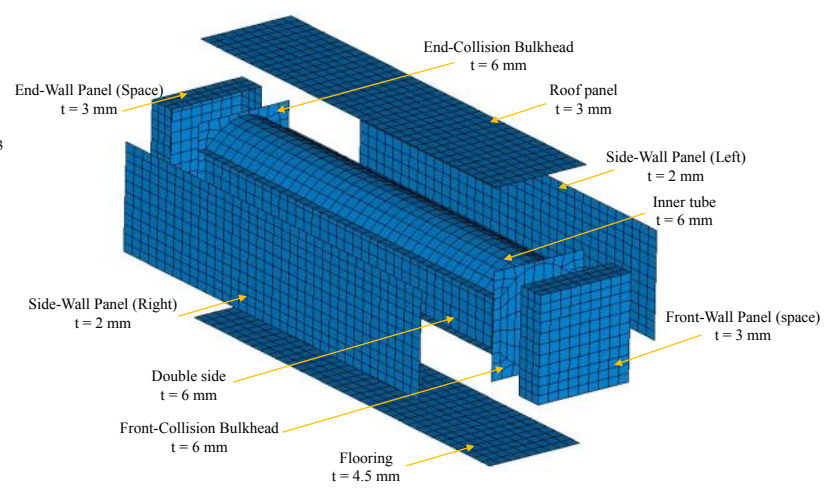

Exploded view of the buffer container

Fig. 2. An alternative design: the buffer container.

In other hand, the container model is generated into two geometries. The first container is denoted as the freight container (Fig. 1). The model is built after the regulation of the Det Norske Veritas (DNV) for a 40-foot-container [4]. The applied material on the container is similar with JIS SPA-H Corten steel according to ISO regulation for 1AAA type cargo container [5]. The properties is given as follow: Density $\rho=7850 \mathrm{~kg} / \mathrm{m}^{3}$, Young's modulus $E=200,000 \mathrm{MPa}$, Poisson's ratio $n=0.3$ and Yield strength $\sigma_{Y}=345 \mathrm{MPa}$.

The second container is denoted as the buffer container (Fig. 2) and described as the modified version of the freight design. An inner tube is added inside the container as a large tank to contain fluid-hazardous cargo. On the both sides of the buffer container, double side is provided with size $200 \mathrm{~mm}$ in width and on front and end walls, $700 \mathrm{~mm}$ safety space is applied. Such containment system is considered as a simplified geometry based on construction of the tanker vessel [6]. The fundamental material properties for this model are similar to the freight container. However, structure of the inner tube was applied by a thicker plate than the outer walls, which a steel with thickness $6 \mathrm{~mm}$ will be applied. Failure criterion of the Peschmann [7] will be appended on both container and ship structure. During collision, the ship is determined to stay on the centerline. The end of the ship model is fixed on the transverse frames and rotational displacement is restrained on the shell plating. Detail illustration of the collision scenario is presented in Fig. 3 and boundary conditions on the ship are applied based on pioneer work in ship collision [8].

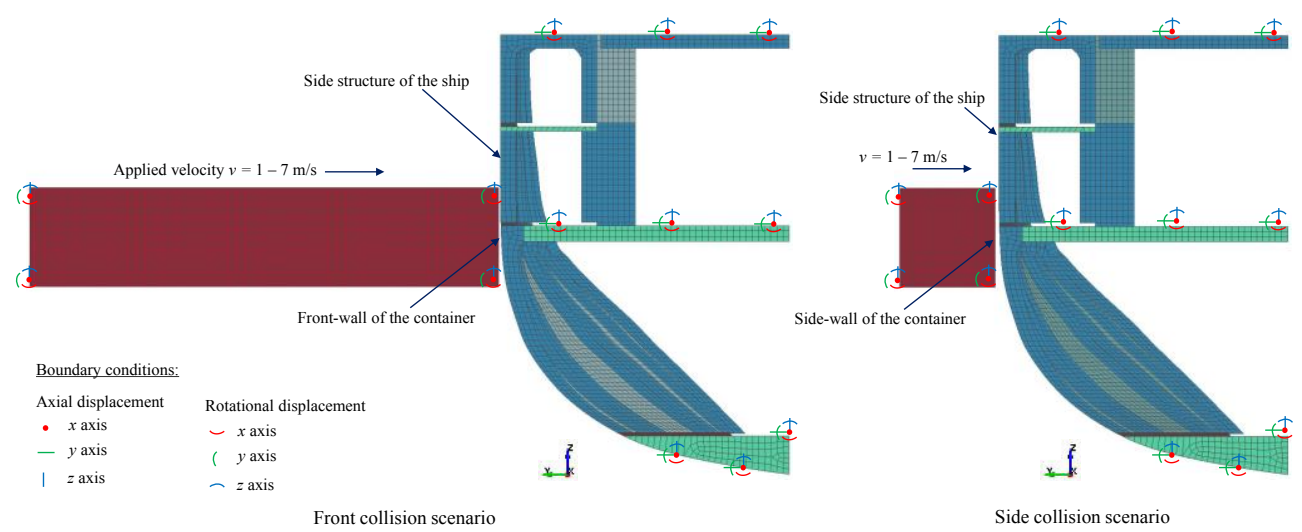

Fig. 3. Scenario for the container collision. Notations highlight boundary conditions on the models. 


\section{Results and Discussion}

The calculation results are presented in terms of the internal energy and other structural responses, such as von Mises stress (effective stress) will be discussed in further parts.

Firstly, the internal energy represents amount of energy which is needed to plastically deform the containers. During collision with the freight container, the energy (Fig. 4) reached peak point and went down after surpassing this point. This result indicates that the container rebounded to opposite of the penetration direction (y axis) after it collided with the side hull. As side part of the container has wider area than the front-wall, the side collision (Fig. 4b) was concluded produce higher energy level than the front collision (Fig. 4a). Besides in terms of energy level, behaviour of two scenarios presented notable distinction. During the front collision, after reaching peak point, the energy reduced and stayed in stable condition. This phenomenon indicated that impulse of the collision (affected by kinetic energy during collision) did not ignite further deformation process when the container experienced rebounding. In other hand, fluctuations were spotted on the side collision especially in $V_{4}=7 \mathrm{~m} / \mathrm{s}$. It is predicted that due to wider impact area than the front collision, collision impulse expanded to wide range and made minor deformation was still experienced by the container in rebounding. Minor was denoted as the fluctuation was not as high as peak point in the first contact. Effect of the collision impulse can be seen in more convenient way during discussion is addressed to the effective stress.

a

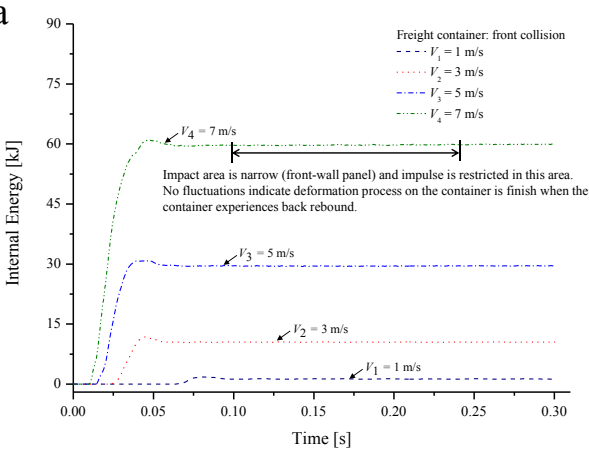

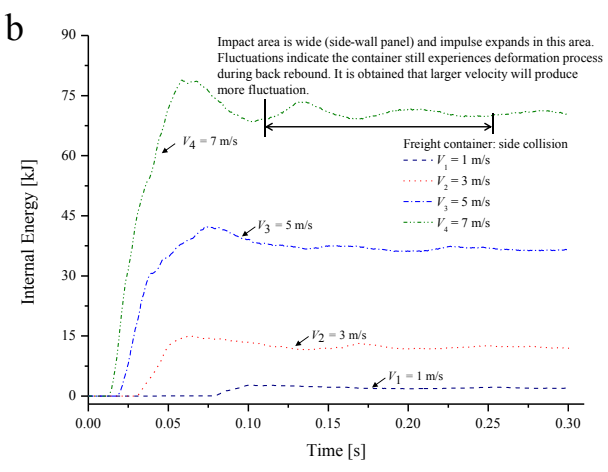

Fig. 4. The internal energy on the freight container: (a) front collision and (b) side collision.
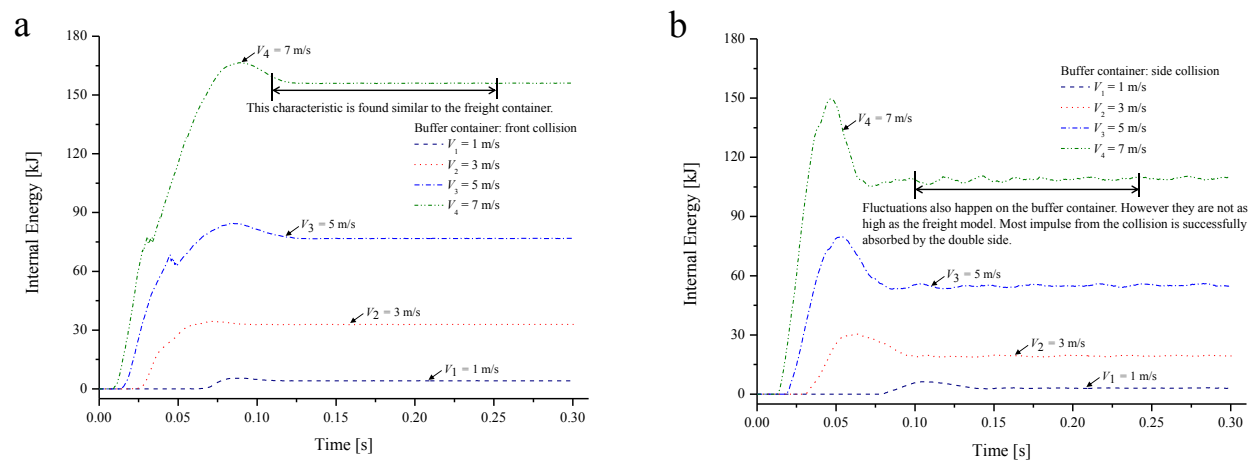

Fig. 5. The internal energy on the buffer container: (a) front collision and (b) side collision.

Next discussion in terms of the internal energy is pointed to the buffer container. As shown in Fig. 5, the buffer design successfully produced higher energy level than the freight container during impact. This result provided a solid indication that resistance capability of a conventional (freight) container had been increased by implementation of 
the double space on side and front panels. It was obtained that the energy increased approximately $160 \%$ in the front collision and more than $85 \%$ in the side collision during the buffer design was implemented on the freight container. Contributions of the double spaces' size was predicted as a major factor in difference of the strength increment percentage. The front panel was applied by larger space than the side space which provided better resistance in collision. The spaces on the buffer design are concluded satisfyingly present adequate safety distance which allowed the outer structure of the container to absorb the impulse as generated by the kinetic energy during collision. Contribution of two designs to the inner cargo condition to be explained in the next part.

In this study, other structural response besides the internal energy is also considered, namely the effective stress (Figs. 6 and 7). Energy-stress relationship (the hardest material to be plastically deformed is expected experience higher stress) is achieved based on these results which the freight container produced lower peak stress than the buffer one. In comparison between the front and side collisions, it was obtained that stress expansion on the side collision was significantly wider than the front scenario. Stress contours have also successfully verified fluctuation behaviour in Figs. 4 and 5. The front collision produced stable condition after peak as the impact area was narrow, deformation during rebounding was unlikely and impulse was limited in this area. It was match with the stress contour that focussed on the front wall (Figs. 6a and 7a). However, the side part of container was wide and stress expanded to wider area (Figs. $6 \mathrm{~b}$ and $7 \mathrm{~b}$ ) than the front collision. Fluctuation happened as during this expansion, impulse ignited minor deformation process on the container and appeared as stress contour in these figures.

a

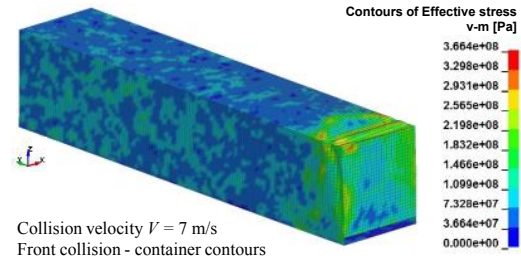

Cont collision - container contours
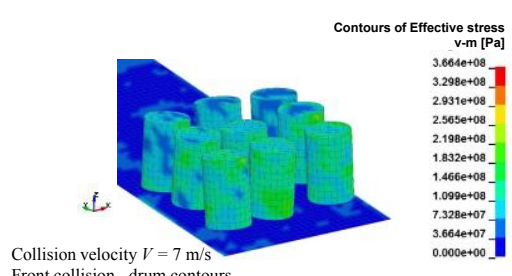

Front collision - drum contours

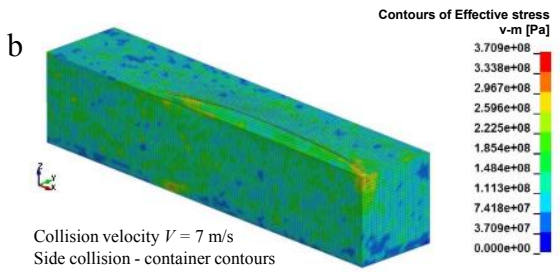

Side collision - container contours

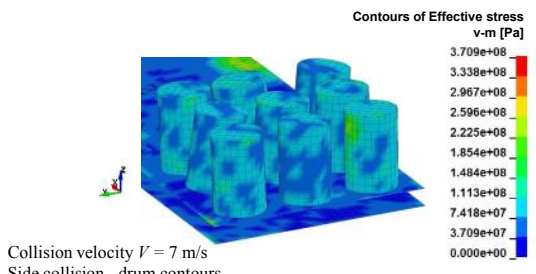

Side collision - drum contours

Fig. 6. Structural response of the freight container collision: (a) front-wall and (b) side-wall.

After that, discussion is directed to the inner container. As shown in Fig. 1, the freight container was assumed to carry fluid cargo using drums, and the buffer container (Fig. 2) was embedded by an inner tube to transport similar cargo. Comparison of the liquid container capacity for both freight and buffer designs was presented in same figures (Figs. 1 and 2). The freight (36 drums - unstacked) could carry liquid cargo approximately 7.8757 $\mathrm{m}^{3}$ or 2080 US liquid gallons and capacity of the buffer design was estimated $144.5232 \mathrm{~m}^{3}$ or equal with more than 38,100 US liquid gallons. Maximum capacity of the freight container was increased $1700 \%$ if the buffer design was applied. Containment system of each container model is found influence structural response under collision. As presented in Fig. 6 that the drums on the freight container which contained fluid cargo were observed collide each other under front and side collisions. Furthermore, contact between the drum and structure was also unavoidable. The drums experienced stress in this situation and 
displaced from its original position. In other situation where belt and plastic are used to ensure fixation on the drums, possibility of displacement is predicted still high and may lead to liquid leakage due to accidental load. Besides its advantage in terms of capacity, effectiveness of the buffer design was also explicitly presented by the stress contour (Fig. 7). As adopted of the double hull concept, stress expansion was reduced under front and side collisions.

a

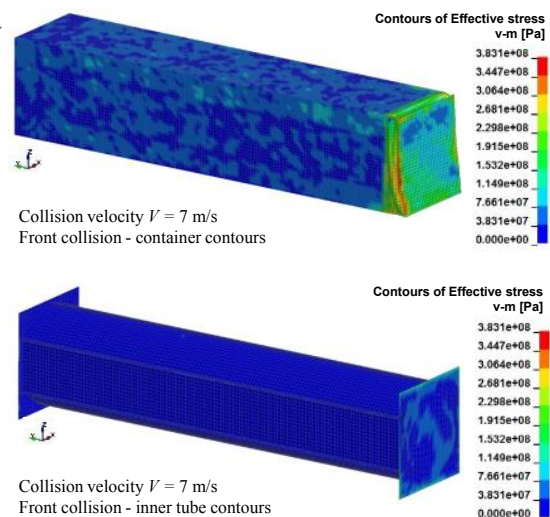

$\mathrm{b}$
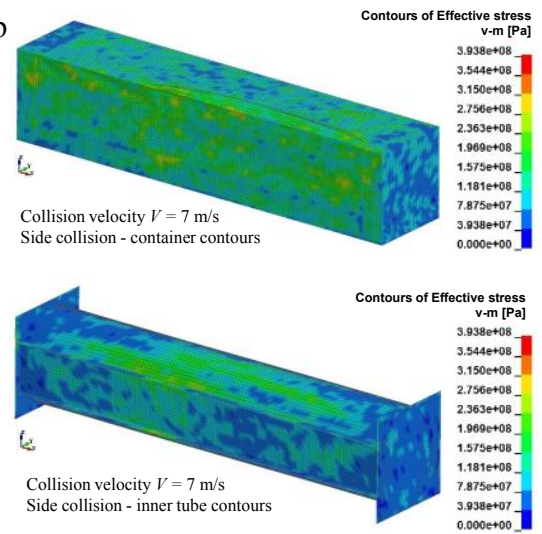

Fig. 7. Structural response after collision to the buffer container: (a) front-wall and (b) side-wall.

\section{Conclusion}

This study aimed to analyse structural responses of containers under collision with other marine structure. It was obtained that the buffer container provided better resistance subjected to impact loads than the freight container. Possibility of cargo leakage was considered unlikely as the safety space provided adequate space to absorb collision impulse. Condition of the inner tube was stable and did not experience displacement as the drums inside the freight container. In damage progression, contribution of the safety space was considered powerful enough to reduce deformation process in rebounding and stress expansion as generated by the kinetic energy after ship-container interaction. This statement was also validated by fluctuation in terms of the internal energy which was almost vanished after the peak point was achieved on the buffer design. Besides offering better structural strength, the buffer design also satisfyingly increases cargo capacity.

\section{Reference}

1. S. Zhang, R. Villavicencio, L. Zhu, P.T. Pedersen, Mar. Struct. 52, 69 (2017)

2. A.R. Prabowo, D.M. Bae, J.M. Sohn, A.F. Zakki, B. Cao, J.H. Cho, Thin-Walled Struct. 115, 225 (2017)

3. A.R. Prabowo, D.M. Bae, J.M. Sohn, A.F. Zakki, B. Cao, Q. Wang, Theo. Appl. Mech. Lett. 7, 6 (2017)

4. DNV, Rules for certification of freight container (Det Norske Veritas, Høvik, 1981)

5. ISO 668, Series 1 freight containers - Classification, external dimensions and ratings (International Organization for Standardization, Geneva, 2013)

6. D.J. Eyres, Ship construction, fifth edition (Butterworth Heinemann, Oxford, 2001)

7. E. Lehmann, J. Peschmann, Mar. Struct. 15, 429 (2002)

8. A.R. Prabowo, S.J. Baek, H.J. Cho, J.H. Byeon, D.M. Bae, J.M. Sohn, Lat. Am. J. Sol. Struct. 14, 1345 (2017) 